\title{
Adoption of Agroforestry Systems by Farmers in Masaka District of Uganda
}

\author{
Vincent B. Sebukyu and D.M. Mosango
}

\section{Research}

\begin{abstract}
High population density in Masaka district, Uganda, has led to increased pressure on land. This has resulted in reduced arable land and decreased soil fertility. In view of this, the Vi-Tree NGO Project has initiated a study to investigate the adoption of agroforestry systems by farmers in Masaka district with focus on the types of agroforestry systems practiced, incorporation of multipurpose tree and shrub species, crops grown, farm land size and land ownership, production and marketing problems, and the main items on which families expend money on. Data was collected using open-ended questionnaire interviews administered to 88 farmers randomly selected throughout the 22 subcounties of Masaka district. The results were analyzed using descriptive statistics. The findings showed that the main agroforestry systems practiced are agrosilvopasture, agrosilviculture and silviculture. The study revealed that 81 woody species (75 trees and 6 shrubs) are used. $69 \%$ of these are indigenous. The most important families are Fabaceae, Moraceae, Euphorbiaceae, Combretaceae and Myrtaceae. Bananas, cassava, beans, vegetables, maize and coffee are the main crops grown. The majority of farmers hold small farm lands (1- 3 acres). Primary production problems are pests and diseases while marketing problems are many including low prices, long distances from village to farm, lack of buyers and price fluctuations. The main items they spend money on are school fees, medicines, and essential commodities. The adoption of agroforestry systems by farmers is relatively high in Masaka district. This is probably due to high demand for land, soil fertility decline, erosion problems, and demand for woody products (e.g., timber, fuel wood, and fodder, food), contact with the Vi-Tree NGO Agroforestry Project extension agents, and need to increase crop yield. High levels of personal land ownership has probably contributed to the adoption of agroforestry systems to pro-
\end{abstract} mote long-term production.

\section{Introduction}

Agroforestry is a dynamic, ecologically based, natural resources management system that, through the integration of trees on farms and in the agricultural landscape, diversifies and sustains production for increased social, economic and environmental benefits for land users at all levels (ICRAF 2006, Kang et al. 1990). Huxley and van Houten (1997) define agroforestry systems as land-use in which woody perennials (trees, shrubs, palms, bamboos) are deliberately used on the same land management unit as agricultural crops (woody or annual), animals or both, in some form of spatial arrangement or temporal sequence.

In Masaka district, Uganda, as in other parts of tropical Africa, local farmers have practiced agricultural systems that encourage the development of forests through fallows as part of sustainable land use. This traditional system is known as the bush fallow system or shifting cultivation. Shifting cultivation is characterized by short cropping

\section{Correspondence}

Vincent, B. Sebukyu, National Organic Agricultural Movement of Uganda, P.O. Box 70071, Kampala, UGANDA

vsebukyu@nogamu.org.ug

D. M. Mosango, Makerere University, Botany Department, P.O. Box 7062, Kampala, UGANDA.

dmbokuyo@yahoo.fr

Ethnobotany Research \& Applications 10:059-068 (2012)

Published: March 29, 2012

www.ethnobotanyjournal.org/vol10/i1547-3465-10-059.pdf 
periods (1-3 years), followed by long fallow periods of 6 or more years. During the fallow period, plant cover and leaf litter protect the soil from the impact of high rain intensity and improve soil fertility. The bush-fallow system was a sustainable and efficient way for restoring soil fertility and productivity, when arable land was abundant and human populations were low. Traditionally, this system used to produce a wide variety of products such as timber, fuel wood, fruits, vegetables, spices, resins, and medicines, primarily to meet household needs and to generate some income through sales in local markets (Roshetko et al. 2006).

Nowadays, due to rapid increase in human populations and subsequent demand for farm land and wood products, long fallow periods (over 10 years) have been shortened drastically. This has led to increased land degradation, increased weed infestation, and decreased food crop yields. Hence, this traditional system is no longer possible due to high pressure on land.

Agroforestry is now emerging as the promising land use option to sustain agricultural productivity and livelihoods of farmers (Syampunani et al. 2010). The new agroforestry system is therefore a sustainable production system (Kang \& Van den Beldt 1990). It has many advantages for farmers. It allows a longer cropping period, more intensive cropping, and higher crop yields (Kang \& Wilson 1987, Mosango 1999). It also allows rapid soil fertility restoration and reduces requirements for external inputs of fertilizer. The hedgerows in the system provide green manure and mulch for crops, biologically fixed nitrogen for companion crops, improve soil conservation, create favorable conditions for beneficial soil organisms, provide high-protein fodder for livestock, and staking material and/or firewood.

This is why farmers in Masaka district have been encouraged by the Vi-Tree non-government organization (NGO) Project to practice agroforestry systems by growing crops with trees or shrubs simultaneously on the same land in order to overcome the reduction of arable land, assure food security and improve their livelihood. For this reason, the Vi-Tree NGO Project has introduced and promoted new agroforestry systems in Masaka district, emphasizing use of a system of alley farming. Thus, fast growing trees or shrubs - preferably woody legume nitrogen-fixing species - have been grown with crops or pastures. These trees are mostly established in the hedgerows with crops or pasture in-between. They are periodically pruned and managed during the cropping phase to prevent shading. The pruning of foliage and young stems are used as green manure or as mulch. Some portion of the tree foliage can be harvested and fed to livestock.

The purpose of this study was to investigate and assess the potential adoption of new agroforestry systems by farmers in Masaka district and their contributions to rural livelihood in terms of multiple land management, farm yield, availability of more products, increase in income and non reliance on fertilizer.

\section{Methods}

\section{Study area}

Masaka district is located in central Uganda. It is bordered in the north by the districts of Mubende and Mpigi, in the west by the districts of Mbarara and Rakai, in the south by the district of Rakai, and in the east by the Lake Victoria. It has an estimated area of $10,611 \mathrm{~km}^{2}$ comprising of 6 counties with 22 sub-counties, including Masaka municipality.

The district has an altitudinal range of 1219-1524 m above sea level, characterized by flat topped hills of uniform height of about $1311 \mathrm{~m}$. The area has a mean annual temperature of $20.8^{\circ} \mathrm{C}$ with a minimum range of $14.5-16.0^{\circ} \mathrm{C}$ and a maximum range of $25.5-27.5^{\circ} \mathrm{C}$. The Masaka district has a population of 836,736 people of which 423,184 are females and 415,550 are males. The population density is 248 persons per $\mathrm{km}^{2}$. The Masaka district has 176,882 households with an average size of 4.3 . The average annual population growth is $2.35 \%$. The people of the area are predominately farmers and their main food crops include bananas, beans, maize, potatoes and cassava. The vegetation is short grass on hill tops and forests in the valleys, giving way to Cyperus papyrus L. in the swamps. Scattered natural forests are found along lakeshores.

\section{Data collection and analysis}

Data were collected from the twenty-two Masaka district subcounties. Farmers were interviewed using an openended questionnaire. The questionnaire sought information on respondents' attitude towards agroforestry systems, the tree/shrub species and crops grown, land size and ownership, production and marketing problems and family main expenditures. In most cases the questions were translated into the local language (Luganda) for the respondents to understand. Woody perennial species used in each agroforestry system were recorded, identified, and brought to the herbarium of Makerere University herbarium (MHU).

Relative prevalence (RP) (Hocking et al. 1996) was calculated for different trees/shrubs used in agroforestry system as a measure of importance. All results obtained were presented and discussed in terms of descriptive statistics.

\section{Results}

\section{Agroforestry systems practiced in Masaka district}

The study of agroforestry practices carried out in Masaka district revealed five types of agroforestry systems (Table 1). Most farmers practice Agrosilvipasture. 


\section{Sebukyu \& Mosango - Adoption of Agroforestry Systems by Farmers in Masaka District of Uganda}

Table 1. Agroforestry systems in Masaka district, Uganda.

\begin{tabular}{|l|c|r|}
\hline Agroforestry systems & Number of farmers & \% \\
\hline Agrosilvipasture & 40 & 45.5 \\
\hline Agrosilviculture & 29 & 32.9 \\
\hline Silvipasture & 14 & 16.0 \\
\hline Apiculture & 4 & 4.5 \\
\hline Agro-aqua-silviculture & 1 & 1.1 \\
\hline Total & 88 & 100 \\
\hline
\end{tabular}

Species and family diversity, importance and relative prevalence

82 tree/shrub species, distributed among 28 families, were found to be grown with agricultural crops (Table 2). $77(93.9 \%)$ of species are trees and $45(54.9 \%)$ of species are indigenous. The dominant plant family is Fabaceae with 19 species (Table 3). The most common indigenous tree species based on PR are and Ficus natalensis Hochst. (RP=5.11) and Markhamia lutea (Benth.)

Table 2. Plant species used in agroforestry systems in Masaka district, Uganda. BF=biological form, St=status, $T=t r e e$, $\mathrm{S}=$ shrub, $\mathrm{E}=$ exotic, I=indigenous. Local names in Luganda

\begin{tabular}{|c|c|c|c|c|c|}
\hline BF & St & Plant species & Local name & Prevalence & Function/Uses \\
\hline & & \multicolumn{4}{|l|}{ ANACARDIACEAE } \\
\hline \multirow[t]{2}{*}{$\mathrm{T}$} & $E$ & Mangifera indica L. & Muyembe & 2.79 & Fruit,shade, forage, firewood \\
\hline & & \multicolumn{4}{|l|}{ APOCYNACEAE } \\
\hline $\mathrm{T}$ & I & Funtumia elastica (Preuss) Stapf & Nkago & 0.26 & $\begin{array}{l}\text { Fuel wood, shade, } \\
\text { rubber [latex] }\end{array}$ \\
\hline \multirow[t]{2}{*}{$\mathrm{T}$} & $E$ & $\begin{array}{l}\text { Thevetia peruviana (Pers.) } \\
\text { K. Schum. }\end{array}$ & None & 0.00 & Tool handles, soil conservation \\
\hline & & \multicolumn{4}{|l|}{ ARALIACEAE } \\
\hline \multirow[t]{2}{*}{$\mathrm{T}$} & 1 & Polyscias fulva (Hiern) Harms & Setala & 0.01 & $\begin{array}{l}\text { Timber, carvings, } \\
\text { mulch, fuel wood }\end{array}$ \\
\hline & & \multicolumn{4}{|l|}{ ASPARAGACEAE } \\
\hline \multirow[t]{2}{*}{$S$} & I & Dracaena fragrans (L.) Ker Gawl. & Oluwaanyi & 0.02 & Boundary mark, hedge \\
\hline & & \multicolumn{4}{|l|}{ ASTERACEAE } \\
\hline$S$ & 1 & Vernonia amygdalina Delile & Mululuuza & 0.00 & Medicinal \\
\hline \multirow[t]{2}{*}{$S$} & I & Vernonia auriculifera Hiern & Kikokooma & 0.03 & Toilet paper, fuel wood \\
\hline & & \multicolumn{4}{|l|}{ BIGNONIACEAE } \\
\hline $\mathrm{T}$ & $E$ & Jacaranda mimosifolia D. Don & Mujakalanda & 0.06 & Fuel wood, timber, bee forage \\
\hline $\mathrm{T}$ & I & $\begin{array}{l}\text { Markhamia lutea (Benth.) } \\
\text { K. Schum. }\end{array}$ & Musembe & 3.18 & Building poles, fuel wood \\
\hline \multirow[t]{2}{*}{$\mathrm{T}$} & 1 & $\begin{array}{l}\text { Spathodea campanulata } \\
\text { P. Beauv. }\end{array}$ & Kifabakazi & 0.03 & Medicinal, fuel wood, timber \\
\hline & & \multicolumn{4}{|l|}{ BORAGINACEAE } \\
\hline \multirow[t]{2}{*}{$\mathrm{T}$} & 1 & Cordia africana Lam. & Mukibi & 0.09 & Fuel wood, building, shading \\
\hline & & \multicolumn{4}{|l|}{ BURSERACEAE } \\
\hline \multirow[t]{2}{*}{$\mathrm{T}$} & 1 & Canarium schweinfurthii Engl. & Muwafu & 0.00 & Fuel wood, building, timber \\
\hline & & \multicolumn{4}{|l|}{ CASUARINACEAE } \\
\hline \multirow[t]{2}{*}{$\mathrm{T}$} & $\mathrm{E}$ & Casuarina equisetifolia L. & Kalivaliyo & 1.30 & Fuel wood \\
\hline & & \multicolumn{4}{|l|}{ CLUSIACEAE } \\
\hline \multirow[t]{2}{*}{$\mathrm{T}$} & 1 & Garcinia buchananii Baker & Nsaali & 0.00 & Poles, building, shade \\
\hline & & \multicolumn{4}{|l|}{ COMBRETACEAE } \\
\hline $\mathrm{T}$ & 1 & $\begin{array}{l}\text { Combretum molle R.Br. } \\
\text { ex G. Don }\end{array}$ & Ndagi & 0.00 & Timber, fuel wood \\
\hline $\mathrm{T}$ & $\mathrm{I}$ & Terminalia brownii Fresen. & Nkalati & 0.02 & Timber, fuel wood \\
\hline
\end{tabular}




\begin{tabular}{|c|c|c|c|c|c|}
\hline $\mathrm{BF}$ & St & Plant species & Local name & Prevalence & Function/Uses \\
\hline $\mathrm{T}$ & 1 & Terminalia glaucescens Planch. & Muyati & 0.01 & Bee hives, charcoal, firewood \\
\hline $\mathrm{T}$ & 1 & Terminalia ivorensis A. Chev. & Muyati & 0.00 & $\begin{array}{l}\begin{array}{l}\text { Fuel wood, charcoal, } \\
\text { timber, coffee shade }\end{array} \\
\end{array}$ \\
\hline \multirow[t]{2}{*}{$\mathrm{T}$} & $E$ & Terminalia mantaly H. Perrier & Muyati & 0.22 & Shade, ornamental \\
\hline & & \multicolumn{4}{|l|}{ EUPHORBIACEAE } \\
\hline $\mathrm{T}$ & $E$ & Aleurites moluccanus (L.) Willd. & Kabakanjagala & 0.00 & Fuel wood, food \& oil [seeds], \\
\hline $\mathrm{T}$ & 1 & $\begin{array}{l}\text { Bridelia micrantha (Hochst.) } \\
\text { Baill. }\end{array}$ & Katazamiti & 0.00 & Building poles, fodder \\
\hline $\mathrm{T}$ & 1 & $\begin{array}{l}\text { Croton macrostachyus Hochst. } \\
\text { ex Delile }\end{array}$ & Musogasoga & 0.08 & $\begin{array}{l}\text { Coffee shade, fuel wood, } \\
\text { fodder }\end{array}$ \\
\hline $\mathrm{T}$ & 1 & Croton megalocarpus Hutch. & Nkulumire & 0.09 & Fuel wood, poles, bee forage \\
\hline $\mathrm{T}$ & 1 & Croton sylvaticus Hochst. & Musogasoga & 0.00 & $\begin{array}{l}\text { Fuel wood, timber, } \\
\text { shade, poles }\end{array}$ \\
\hline $\mathrm{T}$ & $E$ & $\begin{array}{l}\text { Euphorbia tulearensis (Rauh) } \\
\text { Rauh }\end{array}$ & Nkoni & 0.00 & Medicine, fish poison [latex] \\
\hline$S$ & $E$ & Ricinus communis $\mathrm{L}$. & Nsogasoga & 0.00 & Medicine [castor oil], oil [seeds] \\
\hline \multirow[t]{2}{*}{$\mathrm{T}$} & 1 & Sapium ellipticum (Hochst.) Pax & Musasa & 0.11 & $\begin{array}{l}\text { Medicine, tool handles, } \\
\text { fuel wood }\end{array}$ \\
\hline & & \multicolumn{4}{|l|}{ FABACEAE } \\
\hline $\mathrm{T}$ & $E$ & Acacia hockii De Wild. & Kasaana & 0.01 & $\begin{array}{l}\text { Firewood, medicine [roots], } \\
\text { ropes [bark], fencing. }\end{array}$ \\
\hline $\mathrm{T}$ & $E$ & Acacia mearnsii De Wild. & Nsaana & 0.02 & $\begin{array}{l}\text { Firewood, utensils [pestles], } \\
\text { medicine, fodder, nitrogen } \\
\text { fixation. }\end{array}$ \\
\hline $\mathrm{T}$ & 1 & Acacia sieberiana DC. & Mweramanyo & 0.00 & $\begin{array}{l}\text { Firewood, timber, fodder, } \\
\text { tool handles, nitrogen } \\
\text { fixation, gum, fences } \\
\end{array}$ \\
\hline $\mathrm{T}$ & $E$ & Acrocarpus fraxinifolius Arn. & - & 0.00 & Fuel wood, bee forage, shade \\
\hline $\mathrm{T}$ & $E$ & Albizia chinensis (Osbeck) Merr. & Mugavu & 0.00 & Firewood, timber, poles \\
\hline $\mathrm{T}$ & 1 & Albizia coriaria Welw. ex Oliv. & Mugavu & 0.01 & Firewood, timber, poles \\
\hline $\mathrm{T}$ & 1 & Baikiaea insignis Benth. & Nkobakoba & 0.00 & Fuel wood, timber, shade \\
\hline $\mathrm{T}$ & $E$ & Calliandra calothyrsus Meisn. & Kaliyandura & 101.80 & $\begin{array}{l}\text { Fodder, nitrogen fixation, } \\
\text { hedge }\end{array}$ \\
\hline $\mathrm{T}$ & 1 & $\begin{array}{l}\text { Entada abyssinica Steud. } \\
\text { ex A. Rich. }\end{array}$ & Mwoloola & 0.06 & Firewood, medicine \\
\hline $\mathrm{T}$ & 1 & Erythrina abyssinica Lam. & Ejirikiti & 0.00 & $\begin{array}{l}\text { Utensils, bee forage, nitrogen } \\
\text { fixation, live fence, mulch }\end{array}$ \\
\hline $\mathrm{T}$ & $E$ & $\begin{array}{l}\text { Gliricidia sepium (Jacq.) Kunth } \\
\text { ex Walp. }\end{array}$ & Jiliricidiya & 0.30 & $\begin{array}{l}\text { Fuel wood, posts, fodder, } \\
\text { shade, bee forage. }\end{array}$ \\
\hline $\mathrm{T}$ & $E$ & $\begin{array}{l}\text { Leucaena leucocephala } \\
\text { (Lam.) De Wit* }\end{array}$ & Lusiina & 0.22 & $\begin{array}{l}\text { Fodder, fuel wood, mulch, } \\
\text { wind break, nitrogen fixation }\end{array}$ \\
\hline $\mathrm{T}$ & 1 & Mimosa scabrella Benth. & Mimosa & 0.35 & $\begin{array}{l}\text { Fuel wood, mulch, timber, } \\
\text { coffee shade, soil } \\
\text { conservation, nitrogen fixation }\end{array}$ \\
\hline $\mathrm{T}$ & 1 & $\begin{array}{l}\text { Piliostigma thonningii } \\
\text { (Schumach.) Milne-Redh. }\end{array}$ & Bauhinia & 0.01 & Food, fodder, shade, tannin \\
\hline
\end{tabular}




\section{Sebukyu \& Mosango - Adoption of Agroforestry Systems by Farmers in Masaka District of Uganda}

\begin{tabular}{|c|c|c|c|c|c|}
\hline $\mathrm{BF}$ & St & Plant species & Local name & Prevalence & Function/Uses \\
\hline$T$ & $E$ & $\begin{array}{l}\text { Senna siamea (Lam.) } \\
\text { H.S. Irwin \& Barneby }\end{array}$ & Gasiya, Omuzungu & 0.02 & $\begin{array}{l}\text { Medicinal, fuel wood, shade, } \\
\text { soil conservation }\end{array}$ \\
\hline$T$ & $E$ & $\begin{array}{l}\text { Senna spectabilis (D.C.) } \\
\text { H.S.Irwin \& Barneby }\end{array}$ & Gasiya, Omuzungu & 1.05 & $\begin{array}{l}\text { Charcoal, poles, bee keeping, } \\
\text { forage, wind break }\end{array}$ \\
\hline $\mathrm{T}$ & $E$ & Sesbania sesban (L.) Merr. & Muzimbandeya & 14.40 & $\begin{array}{l}\text { Nitrogen fixation, shade, soap, } \\
\text { fodder, fuel wood }\end{array}$ \\
\hline $\mathrm{T}$ & $E$ & Tamarindus indica L. & Mukoke & 0.02 & Fruit, fuel wood \\
\hline \multirow[t]{2}{*}{$S$} & $\mathrm{I}$ & Tephrosia vogelii Hook.f. & Muluku & 0.03 & Pesticide \\
\hline & & \multicolumn{4}{|l|}{ LAURACEAE } \\
\hline $\mathrm{T}$ & $E$ & Cinnamomum verum J. Presl & Mudalasiini & 0.00 & Fuel wood, spice for tea \\
\hline$T$ & $E$ & Persea americana Mill. & Ovakaddo & 0.84 & Fruit \\
\hline \multirow[t]{2}{*}{$\mathrm{T}$} & 1 & $\begin{array}{l}\text { Beilschmiedia ugandensis } \\
\text { Rendle }\end{array}$ & Mwasa & 0.00 & Fuel wood, timber, food. \\
\hline & & \multicolumn{4}{|l|}{ MELIACEAE } \\
\hline $\mathrm{T}$ & $E$ & Azadirachta indica A. Juss. & Nnimu & 0.02 & Medicinal \\
\hline$T$ & 1 & Khaya anthotheca (Welw.) C.DC. & Munyanya & 0.00 & Fuel wood, timber, shade \\
\hline \multirow[t]{2}{*}{$T$} & $E$ & Melia azedarach $\mathrm{L}$. & Mutankuyege & 2.28 & $\begin{array}{l}\text { Fuel wood, timber, poles, } \\
\text { medicine, wind break }\end{array}$ \\
\hline & & \multicolumn{4}{|l|}{ MORACEAE } \\
\hline $\mathrm{T}$ & 1 & Antiaris toxicaria Lesch. & Kirundu & 0.00 & Poles, fuel wood \\
\hline$T$ & $E$ & Artocarpus heterophyllus Lam. & Kifenensi & 1.85 & $\begin{array}{l}\text { Firewood, food, shade, lorry } \\
\text { bodies }\end{array}$ \\
\hline$T$ & $E$ & Ficus elastica Roxb. ex Hornem. & Para & 0.00 & $\begin{array}{l}\text { Firewood, shade, avenue } \\
\text { ornamental }\end{array}$ \\
\hline $\mathrm{T}$ & $\mathrm{I}$ & Ficus maitin Pittier & Muserere & 0.09 & \\
\hline$T$ & $\mathrm{I}$ & Ficus mucuso Welw. ex Ficalho & Kabalira & 0.00 & Fuel wood, timber, carving \\
\hline $\mathrm{T}$ & 1 & Ficus natalensis Hochst. & Mutuba & 5.11 & $\begin{array}{l}\text { Medicine, shade, fencing, } \\
\text { bark cloth }\end{array}$ \\
\hline $\mathrm{T}$ & 1 & Ficus ovata Vahl & Kookwe & 0.00 & $\begin{array}{l}\text { Poles, shade, soil } \\
\text { conservation, fence, bark cloth }\end{array}$ \\
\hline$T$ & 1 & Ficus sur Forssk. & Kabalira & 0.00 & $\begin{array}{l}\text { Timber, mortars, beer, canoes, } \\
\text { food, ceremonial }\end{array}$ \\
\hline$T$ & 1 & Ficus sycomorus L. & Luwawu & 0.00 & $\begin{array}{l}\text { Canoe timber, sand } \\
\text { paper [leaves] }\end{array}$ \\
\hline $\mathrm{T}$ & 1 & Milicia excelsa (Welw.) C.C. Berg & Muvule & 0.08 & $\begin{array}{l}\text { Firewood, timber, shade, } \\
\text { mulch, ornamental }\end{array}$ \\
\hline \multirow[t]{2}{*}{$T$} & $E$ & Morus alba L. & Nkenene & 0.40 & $\begin{array}{l}\text { Firewood, food, fodder, } \\
\text { windbreak, fencing, shade }\end{array}$ \\
\hline & & \multicolumn{4}{|l|}{ MYRISTICACEAE } \\
\hline \multirow[t]{2}{*}{$T$} & 1 & $\begin{array}{l}\text { Pycnanthus angolensis } \\
\text { (Welw.) Warb. }\end{array}$ & Munaaba & 0.00 & $\begin{array}{l}\text { Shade, ornamental, } \\
\text { soap, illuminant. }\end{array}$ \\
\hline & & \multicolumn{4}{|l|}{ MYRTACEAE } \\
\hline$T$ & $E$ & $\begin{array}{l}\text { Callistemon citrinus } \\
\text { (Curtis) Skeels. }\end{array}$ & Nyambaddezitonya & 0.13 & $\begin{array}{l}\text { Fuel wood, medicinal, bee } \\
\text { forage, wind break. }\end{array}$ \\
\hline $\mathrm{T}$ & $E$ & Eucalyptus globulus Labill. & Kalitunsi & 0.09 & Poles, fuel wood, timber \\
\hline
\end{tabular}




\begin{tabular}{|c|c|c|c|c|c|}
\hline BF & St & Plant species & Local name & Prevalence & Function/Uses \\
\hline $\mathrm{T}$ & $E$ & Eucalyptus grandis W. Hill & Kalitunsi & 0.14 & Poles, fuel wood, timber \\
\hline $\mathrm{T}$ & $E$ & Psidium guajava L. & Peera & 0.47 & Fruit, fuel wood \\
\hline \multirow[t]{2}{*}{$\mathrm{T}$} & $\mathrm{E}$ & Syzygium cumini (L.) Skeels & Jambula & 0.01 & Fuel wood, timber, food \\
\hline & & \multicolumn{4}{|l|}{ PHYLLANTHACEAE } \\
\hline \multirow[t]{2}{*}{$\mathrm{T}$} & 1 & Uapaca guineensis Müll.Arg. & Munamagulu & 0.00 & Fuel wood \\
\hline & & \multicolumn{4}{|l|}{ PODOCARPACEAE } \\
\hline \multirow[t]{2}{*}{$\mathrm{T}$} & 1 & $\begin{array}{l}\text { Podocarpus latifolius (Thunb.) } \\
\text { R. Br. ex Mirb. }\end{array}$ & Musenene & 0.19 & Fuel wood, timber, shade \\
\hline & & \multicolumn{4}{|l|}{ PROTEACEAE } \\
\hline \multirow[t]{2}{*}{$\mathrm{T}$} & $E$ & $\begin{array}{l}\text { Grevillea robusta A. Cunn. } \\
\text { ex R.Br. }\end{array}$ & Kalwenda & 149.30 & $\begin{array}{l}\text { Coffee shade, fuel wood, } \\
\text { building material }\end{array}$ \\
\hline & & \multicolumn{4}{|l|}{ PUNICACEAE } \\
\hline \multirow[t]{2}{*}{$\mathrm{T}$} & $E$ & Punica granatum $\mathrm{L}$. & Nkomamawanga & 0.02 & Food, fence, \\
\hline & & \multicolumn{4}{|l|}{ RHAMNACEAE } \\
\hline \multirow[t]{2}{*}{$\mathrm{T}$} & 1 & Maesopsis eminii Engl. & Musizi & 3.90 & Fuel wood, fodder, shade, \\
\hline & & \multicolumn{4}{|l|}{ ROSACEAE } \\
\hline \multirow[t]{2}{*}{$\mathrm{T}$} & $E$ & $\begin{array}{l}\text { Eriobotrya japonica (Thunb.) } \\
\text { Lindl. }\end{array}$ & & 0.10 & $\begin{array}{l}\text { Fruit, Building material, fuel } \\
\text { wood }\end{array}$ \\
\hline & & \multicolumn{4}{|l|}{ RUBIACEAE } \\
\hline \multirow[t]{2}{*}{$\mathrm{T}$} & 1 & Vangueria apiculata K. Schum. & Matuggunda & 0.01 & Firewood, poles, food \\
\hline & & \multicolumn{4}{|l|}{ RUTACEAE } \\
\hline $\mathrm{T}$ & $E$ & Citrus limon (L.) Osbeck & Ennimu & 0.01 & Fruit \\
\hline $\mathrm{T}$ & $E$ & Citrus reticulata Blanco & Mangadda & 0.00 & Fruit \\
\hline $\mathrm{T}$ & $\mathrm{E}$ & Citrus sinensis (L.) Osbeck & Mucungwa & 0.00 & Fruit \\
\hline \multirow[t]{2}{*}{$\mathrm{T}$} & 1 & Teclea nobilis Delile & Enzo & 0.00 & Building material, fuel wood \\
\hline & & \multicolumn{4}{|l|}{ SALICACEAE } \\
\hline \multirow[t]{2}{*}{$\mathrm{T}$} & 1 & $\begin{array}{l}\text { Dovyalis caffra (Hook.f. \& Harv.) } \\
\text { Warb. }\end{array}$ & - & 0.16 & $\begin{array}{l}\text { Fruit jam, live fence, } \\
\text { ornamental }\end{array}$ \\
\hline & & \multicolumn{4}{|l|}{ SOLANACEAE } \\
\hline$S$ & 1 & $\begin{array}{l}\text { Cyphomandra betacea (Cav.) } \\
\text { Sendt. }\end{array}$ & Munyanya & 0.10 & Food [jam, fruit, vegetable] \\
\hline
\end{tabular}

Table 3. The most important plant families in Masaka district, Uganda.

\begin{tabular}{|l|c|r|}
\hline Family & Number of species & $\begin{array}{r}\text { \% } \\
(\mathbf{N}=\mathbf{8 1})\end{array}$ \\
\hline Fabaceae & 19 & 23.4 \\
\hline Moraceae & 11 & 13.6 \\
\hline Euphorbiaceae & 8 & 9.9 \\
\hline Combretaceae & 5 & 6.2 \\
\hline Myrtaceae & 5 & 6.2 \\
\hline Rutaceae & 4 & 4.9 \\
\hline
\end{tabular}

K. Schum. (RP=3.18). The most common exotic species are Grevillea robusta A. Cunn. ex R.Br. (RP=149.3) (from Australia) and Calliandra calothyrsus Meisn. ( $R P=101.8)$ (from Central America). Many exotic tree species are woody legumes used to fix nitrogen.

\section{Crops grown with trees and agroforestry commodities}

Table 4 shows that the most prevalent crop grown with trees is banana $(65.9 \%$ ). (It is the major food crop for Baganda people living in Masaka district.) This is followed by cassava grown by $13.6 \%$ of farmers. (Cassava is used as a food and harvested when there are no mature banana fruits in the farm.) A few farmers in Masaka district $(5.7 \%)$ do not grow crops with trees but practice the older farming system of bush fallow. 


\section{Sebukyu \& Mosango - Adoption of Agroforestry Systems by Farmers in Masaka District of Uganda}

Table 4. Crops grown with trees in Masaka district, Uganda.

\begin{tabular}{|l|c|r|}
\hline Crops & Number of farmers & $\%$ \\
\hline Banana & 58 & 65.9 \\
\hline Cassava & 12 & 13.6 \\
\hline Beans & 4 & 4.5 \\
\hline Maize & 4 & 4.5 \\
\hline Vegetables & 3 & 3.4 \\
\hline Coffee & 2 & 2.3 \\
\hline None & 5 & 5.7 \\
\hline Total & 88 & 100.0 \\
\hline
\end{tabular}

\section{Land size and sources of land for farms}

Many farmers (43\%) have small land areas which range from 1 to 3 acres (0.4-1.2ha) (Table 5).

Table 5. The size of land in the agroforestry systems in Masaka district, Uganda.

\begin{tabular}{|l|c|r|}
\hline $\begin{array}{l}\text { Land size } \\
\text { (acres) }\end{array}$ & Number of farmers & $\%$ \\
\hline $1-3$ & 43 & 48.9 \\
\hline $4-6$ & 32 & 36.4 \\
\hline $7-9$ & 6 & 6.8 \\
\hline 10 & 7 & 7.9 \\
\hline Total & 88 & 100.0 \\
\hline
\end{tabular}

The most common land tenure for farmers $(70.5 \%)$ is personal ownership (Table 6).

Table 6. Land ownership in Masaka district, Uganda.

\begin{tabular}{|l|c|r|}
\hline Land ownership & Number of farmers & $\%$ \\
\hline Personal & 62 & 70.5 \\
\hline Borrowed & 18 & 20.4 \\
\hline Rented & 6 & 6.8 \\
\hline Communal & 2 & 2.3 \\
\hline Total & 88 & 100 \\
\hline
\end{tabular}

\section{Production and marketing problems}

The main production problems faced by the farmers in Masaka district are pests and diseases (13.6\%) (Table 7). However, many farmers $(39.8 \%)$ report no production problems. In contrast, one third of farmers face at least three of the production problems listed.
Table 7. Production problems in Masaka district, Uganda.

\begin{tabular}{|l|c|r|}
\hline Problems & Number of farmers & $\%$ \\
\hline $\begin{array}{l}\text { At least } 3 \\
\text { problems }\end{array}$ & 30 & 34.1 \\
\hline Pests/diseases & 12 & 13.6 \\
\hline Termites & 5 & 5.7 \\
\hline Soil erosion & 3 & 3.4 \\
\hline Long distance & 2 & 2.3 \\
\hline Fertilizers & 1 & 1.1 \\
\hline No problem & 35 & 39.8 \\
\hline Total & 88 & 100 \\
\hline
\end{tabular}

The principal marketing problems are similar in scale including low prices, long distance from village to farm, lack of buyers, and price fluctuations (Table 8). However $25 \%$ of farmers did not report having any marketing problem.

Table 8. Marketing problems in Masaka district, Uganda.

\begin{tabular}{|l|c|r|}
\hline Problems & Number of farmers & $\%$ \\
\hline Low prices & 18 & 20.5 \\
\hline Long distance & 17 & 19.3 \\
\hline Lack of buyers & 14 & 15.9 \\
\hline Price fluctuations & 13 & 14.8 \\
\hline Lack of storage & 4 & 4.5 \\
\hline No problem & 22 & 25.0 \\
\hline Total & 88 & 100.0 \\
\hline
\end{tabular}

\section{Farmers' family expenditure}

The main items farmers spend money on are school fees, medicines, and essential commodities (Table 9).

Table 9. Family expenditure in Masaka district, Uganda.

\begin{tabular}{|l|c|r|}
\hline Expenditure & Number of farmers & $\%$ \\
\hline School fees & 49 & 55.7 \\
\hline Medicine & 19 & 21.6 \\
\hline $\begin{array}{l}\text { Essential com- } \\
\text { modities }\end{array}$ & 19 & 21.6 \\
\hline Transport & 1 & 1.1 \\
\hline Total & 88 & 100 \\
\hline
\end{tabular}

\section{Discussion}

The results indicate that farmers practice different types of agroforestry systems in Masaka district while some farmers practice specialized agroforestry systems (Young 1989), namely apiculture and agro-aquaculture Nair (1989). This shows that the farmers of Masaka dis- 
trict are involved in the multiple land use management. This implies a mix of crops, trees/shrubs, fish and animals. This allows farmers to produce a variety of crop products (see Table 2).

Farmers in Masaka district hold small land sizes (0.41.2ha). Franzel et al. (2001) found in Zambia that farmers also hold comparable land size ( 0.36 ha) and Thangala et al. (2008) report comparable average land holdings in Zimbabwe (0.43-0.6 ha) and Malawi (0.5-1.1 ha). According to these authors, the total land used by small holder farmers practicing agroforestry is on average less than a hectare since they produce mostly for home consumption and sell their produce individually on-farm or at local markets. Despite small arable land size, they get some revenue from the agroforestry products sold. Agea et al. (2005) came to similar conclusions in Mukono district, Uganda.

Despite the fact that farmers hold small arable lands, they are able to increase farm yield by producing a variety of crops, livestock and wood products for both home consumption and local markets. In spite of this, they do face some production and marketing problems. Pest, diseases and termites negatively affect crop production as they reduce crop yield. Most farmers are poor and cannot afford fertilizers, and have farms far from where they live. Pest and diseases, soil erosion, long distance from the village to farm and termites are the main limiting factors for crop production cited by farmers.

Four marketing problems (low prices, price fluctuations, lack of storage, long distance to the market, and lack of buyers at home level) are comparable to results in Mukono district (Agea et al. 2005) where the physical nature of the product, handling and lack of transport and storage facilities present serious marketing problems. According to Ngategize and Kaboyo (2001), the availability of good transport and storage facilities are key determinants of good price for farmers' products. According to Agea et al. (2005), selling of the produce individually and locally does not offer better income to farmers because local buyers offer only low prices.

Land tenure plays a great role in agroforestry adoption. In Masaka district, personal ownership (divided inheritance or purchased) is the most prevalent category. This type of land tenure provides long-term security that is required for agroforestry adoption (Suyanto et al. 2005). This likely explains why the majority of farmers in Masaka district are involved in agroforestry. Farmers renting land do not practice agroforestry because they hold land for a short time.

The study revealed a diversity of trees and shrubs involved in the Masaka district agroforestry systems. More than half are indigenous but the exotic species are much more preferred. As reported in literature (Kebebew et al. 2011), woody species grown with crops in agroforestry have many advantages. They are known to restore and sustain soil fertility through nutrient cycling, provide mulch and fodder for livestock, and reduce soil erosion. Moreover, woody legumes used in agroforestry replace fallow with continuous cropping, and hence reduce pressure on land by minimizing demand for arable land.

The study showed that farmers' adoption of agroforestry systems is relatively high in Masaka district. The adoption of agroforestry systems by farmers has been made possible by several factors, namely high demand for land due to increasing population, soil fertility decline, erosion problems, and demand for woody products such as fuel wood and fodder, increased crop yield, and contact with the ViTree NGO Agroforestry Project extension. Personal land ownership has also facilitated the adoption of agroforestry systems in Masaka district since majority of farmers hold personal land. In contrast, other types of land ownership may hinder the adoption of agroforestry systems, for example rented or borrowed lands, because farmers cannot use the land for long-term production. This has been pointed out in West and Central Africa countries, Benin, Nigeria, and Cameroon. Thangata et al. (2008) concluded that the potential adoption of agroforestry by farmers in Malawi, Zimbabwe and Zambia depends on household composition, farm size, availability of draft power, and a seed selling incentive. However, in Cameroon, Nkamleu and Manyong (2005) found that the gender of farmer, household family size, level of education, farmer's experience, membership within farmers' associations, contact with research and extension, security of land tenure, agroecological zone, and distance of the village from nearest town, village accessibility and income from livestock facilitate the adoption of agroforestry systems.

\section{Conclusion and Recommendation}

The potential adoption of agroforestry in Masaka district is seen as a means to improve food security and farmers' livelihood. Among factors facilitating this adoption is personal land ownership. Although all the trees and shrubs grown with crops have potential role in agroforestry systems, the woody leguminous species are the most important group because of their economic uses and ecological adaptability (Nair et al. 1984, Mosango 1999). In fact, woody leguminous species have the added advantage because of their capability for nitrogen fixation. Hence, replacing the less productive woody species with fast growing nitrogen fixing species, such as Leucaena leucocephala (Lam.) De Wit, C. calothyrsus, Sesbania sesban (L.) Merr., Gliricidia sepium (Jacq.) Kunth ex Walp., Erythrina abyssinica Lam., and Mimosa scabrella Benth., will increase crop yield, provide farmers with more fuel, fodder and green manure (see Table 2) and improve farmer's livelihood.

Most of the leguminous species used were introduced by the Vi-Tree NGO Project. It is therefore important to select and test several local legume species (Table 2) to test 


\section{Sebukyu \& Mosango - Adoption of Agroforestry Systems by Farmers in Masaka District of Uganda}

for improved agroforestry practices. Moreover, trees with a low relative prevalence should be planted around Masaka district in numerous uninhabited areas and forest reserves so that they can be saved from possible extinction and used later when need arises.

Finally, in order to meet the demand for food and for other wood products in Masaka district, farmers should be encouraged to practice agroforestry systems. The implementation of improved agroforestry systems allows alleviating pressure on natural forests, increasing crop yield, providing farmers with more income and improving their livelihood (Russo 1996).

\section{Acknowledgements}

Our thanks go to the staff of the Makerere University, Botany Department and of the Vi-Tree NGO Project Development at Masaka district and to the farmers of Masaka district and the anonymous reviewers for their very helpful collaboration.

\section{Literature Cited}

Agea J.G., E. Ssebuliba \& B. Obaa. 2005. Marketing of agroforestry products in Nama sub-county, Mukono district, Uganda. African Crop Science Proceedings 7:537540 .

Franzel, S., R. Coe, P. Cooper, F. Place \& S.J. Sherr. 2001. Assessing the adoption potential of agroforestry practices in Sub-Saharan Africa. Agricultural Systems 69(1-2):37-62.

Hocking, D., A. Hocking \& I. Khairu. 1996. Trees on farms in Bangladesh (3): Farmers' species preferences for homestead trees, Survival of new tree planting, and main causes of tree death. Agroforestry Systems 33:231-247.

Huxley, P. \& H. van Houten. 1997. Glossary for Agroforestry. International Centre for Research in Agroforestry (ICRAF), Nairobi, Kenya.

ICRAF. 2006. International Centre for Research in Agroforestry. World Agroforestry Centre, Southeast Asia web site. www.worldagroforestrycentre.org/sea. Date accessed: 03 June 2011.

Kang, B.T. \& G.F. Wilson, 1987. The development of alley cropping as a promising agroforestry technology. Pp. 227243 in Agroforestry: A decade of development. Edited by H.A. Steppler \& P.K.R. Nair. International Centre for Research in Agroforestry, Nairobi, Kenya.

Kang, B.T. \& R.J. Van den Beldt. 1990. Agroforestry systems for sustained crop production in the tropics, with special reference to West Africa. Pp 13-33 in Agroforest- ry land-use systems: Proceedings of a special session on agroforestry land-use systems. American Society of Agronomy Annual Meeting, International Agronomy Section, 28-29 November 1988. Anaheim, California. Edited by E. Moore. Nitrogen Fixing Tree Association, Waimanalo, Hawai i.

Kang, B.T., Reynolds, L. \& A.N. Atta-Krah. 1990. Alley farming. Advances in Agronomy 43:315-359.

Kebebew, Z. \& K. Urgessa. 2011. Agroforestry perspective in land use and farmers coping strategy: Experience from Southwestern Ethiopia. World Journal of Agricultural Sciences 7(1):73-77.

Mosango, M. 1999. Chemical characteristics of six woody species for alley cropping. Tropicultura 17:93-95.

Nair, P.K.R. 1989. Classification of agroforestry systems, Pp. 39-52 in Agroforestry System in the Tropics. Kluwer Boston, Massachusetts.

Nair, P.K.R., E.C Fernandes \& P.N. Wambugu. 1984. Multipurpose leguminous trees and shrubs for agroforestry. Agroforestry Systems 2:145-163.

Ngategize, P.K. \& G. Kaboyo. 2001. Agricultural marketing. Pp.242-271 in Agriculture in Uganda. Edited by J.K. Mkubi. Fountain Publishers Ltd, Kampala, Uganda.

Nkamleu, G.B. \& V. M. Manyong. 2005. Factors affecting the adoption of agroforestry practices by factors in Cameroon. Small-Scale Forestry 4/2:135-148.

Roshetko, J.M., E. Nugraha, J.C.M. Tukan, G. Manurung, C. Fay \& M. van Noordwijk. Agroforestry for livelihood and Enterprise development. www.worldagroforestry.org/ downloads/publications/PDFs/pp07395.pdf Accessed: 03 June 2011

Russo, R.O. 1996. Agrosilvopastoral systems: A practical approach toward sustainable agriculture. Journal of Sustainable Agriculture 7(4):5-16

Suyanto, S., R.P. Pennana, N. Khususiyah \& L. Joshi. 2005. Land tenure, agroforestry adoption, and reduction of fire hazard in a forest zone: A case study from Lampung, Sumatra, Indonesia. Agroforestry Systems 65(1): $1-11$.

Syampunani, S., P.W. Chirwa, F.K. Akinnifest \& O.C. Ajayi. 2010. The potential of using agroforestry as a winwin solution to climate change mitigation and adaptation and meeting food security challenges in Southern Africa. Agricultural Journal 5:80-88.

Thangata, P., M. Mudhara, C. Grier \& P. Hildebrand. 2008 Potential for agroforestry adoption in Southern Africa: A 
comparative study of improved fallow and green manure

Young, A. 1989. Agroforestry for Soil Conservation. C.A.B. adoption in Malawi, Zambia and Zimbabwe. Ethnobotany International, Wallingford, United Kingdom.

Research \& Applications 5:67-75. 